\title{
Patient compliance and periodontal outcomes
}

\section{Abstracted from Lee CT, Huang HY, Sun TC, Karimbux N.}

Impact of Patient Compliance on Tooth Loss during Supportive Periodontal Therapy: A Systematic Review and Meta-analysis. J Dent Res. 2015; 94: 777-786 pii: 0022034515578910. [Epub ahead of print] Review. PubMed PMID: 25818586.

Address for correspondence: N Karimbux, Tufts University School of Dental Medicine, One Kneeland Street, DHS-1512, Boston, MA 02111, USA. E-mail: nadeem.karimbux@tufts.edu

\section{Question: Does a patient's degree of compliance with appointments affect the risk of tooth loss during supportive periodontal treatment?}

Data sources PubMed, Embase and Cochrane Central Register of Controlled Trials (CENTRAL) were searched until 2014. Reference lists from the articles were examined

Study selection Two authors independently searched for randomised and non-randomised controlled trials and prospective and retrospective cohorts with a mean follow up period of at least five years.

Data extraction and synthesis Quality of the studies was assessed using the Newcastle-Ottawa scale (NOS) and the data were extracted on a specially designed form. The primary outcome was calculated as risk ratio of tooth loss (RRTL). Risk difference of tooth loss (RDTL) and weighted mean difference of tooth loss rate (WDTLR) were also calculated.

Results One prospective and seven retrospective cohort studies including 1409 participants were included. The pooled RRTL was calculated as $0.56(\mathrm{Cl}: 0.38,0.82) \mathrm{P}<0.01$, while the pooled RDTL was calculated as $-0.05(\mathrm{Cl}:-0.08,-0.01) \mathrm{p}<0.01$. Based on the risk difference, the NNT was calculated as 20.

Conclusions Tooth loss rate was significantly lower in the regular compliance group over the five years. To prevent one extraction, 20 teeth have to be maintained with regular compliance for more than five years.

\section{Commentary}

Compliance is a subjective concept and the periodontal therapy and its maintenance success depends on it. As previously cited, 'noncompliance to periodontal maintenance cannot be solely explained by one determinant but rather may involve an individual's health beliefs, emotional intelligence, psychological stressors, and personality traits,' compliance is a multifactorial concept. ${ }^{1}$

The authors of this systematic review aimed to analyse the effect of appointment compliance during supportive periodontal therapy (SPT) on tooth loss and to investigate the potential parameters affecting the association between compliance and tooth loss. They intended to include randomised and non-randomised trials, prospective and retrospectives cohorts with at least five years follow-up.

The authors searched three databases restricted to studies in English and did some hand searching. There is no mention of the grey literature search. Two authors independently evaluated for inclusion with a high degree of agreement. Eight studies meeting the inclusion criteria were used in the data analysis: one prospective cohort $(n=265)$ and seven retrospective cohorts $(n=1311)$. The same authors performed data extraction. Original authors were contacted for missing or unclear information.

The quality was assessed using an appropriate tool for observational studies (Newcastle-Ottawa Scale) and it was calculated as high. As the authors mentioned, the dropout rates were barely reported and only one study declared no loss of patients. The authors also identified other possible sources of bias and confounders in the included studies that may limit the generalisation of the results: patient selection and willingness to participate, lack of consistency to determine the periodontal status, partial information on smoking, diabetes or other systemic diseases' presence.

The results were pooled into three meta-analyses. Tooth loss, the primary outcome, was examined as a risk ratio and risk difference. The RR was 0.56 (CI: $0.38,0.82$ ), $\mathrm{p}<0.01$ for the intervention and the risk difference was -0.05 (CI: $-0.08,-0.01) \mathrm{p}<0.01$. The heterogeneity was very high. Based on the risk difference value the number need to treat (NNT) was calculated as 20. The calculated tooth loss ratio also gave an advantage to the intervention.

It is important to mention that the compliance group, in most of the included studies, represented $10 \%$ of the sample size of the studies. This number is lower when compared with the standard rate of absolute compliance in the periodontal field, $32 \%{ }^{2}$

Publication bias was assessed; the symmetric funnel plots presented a low risk of bias. However, following previous 
publication $^{3}$ and Cochrane recommendation, ${ }^{4}$ funnel plots should not examine intervention studies. As well, the Cochrane guidelines recommend funnel plot asymmetry on systematic reviews with at least ten included studies because a reduced number of studies could lower the power of the test to distinguish real asymmetry.

The authors concluded that compliance affects the risk of tooth loss, but the methodological limitations prevent the drawing of conclusions about the magnitude of treatment effect. The cost-benefit for the patients is positive, meaning that by keeping their natural teeth they will spend less money than if teeth were lost.
Camila Pachêco-Pereira a and Silvia Spivakovskyb aschool of Dentistry, Faculty of Medicine and Dentistry, School of Dentistry, University of Alberta, Canada ${ }^{b}$ New York University College of Dentistry, New York, USA

1. The psychology of patient compliance: a focused review of the literature. Umaki TM, Umaki MR, Cobb CM. J Periodontol 2012; 83: 395-400.

2. The results of efforts to improve compliance with supportive periodontal treatment in a private practice. Wilson TG Ir, Hale S, Temple R. / Periodontol 1993; 64: 311-314.

3. Publication bias is underreported in systematic reviews published in high-impactfactor journals: metaepidemiologic study. Onishi A, Furukawa TA. J Clin Epidemiol. 2014; 67: 1320-1326.

4. Addressing reporting biases. J. Higgins, S. Green (Eds.), Cochrane handbook for systematic reviews of intervention. Version 5.1.0 (updated March 2011), New York: The Cochrane Collaboration (2011) [Chapter 10]. Available at http://handbook.cochrane.org/

Evidence-Based Dentistry (2016) 17, 21-22. doi:10.1038/sj.ebd.6401153 